\title{
In-situ calibration of clinical built-in KAP meters with traceability to a primary standard using a reference KAP meter
}

\author{
Alexandr Malusek, Ebba Helmrot, Michael Sandborg, J-E Grindborg and Gudrun Alm \\ Carlsson
}

\section{Linköping University Post Print}

\section{Tweet}

N.B.: When citing this work, cite the original article.

Original Publication:

Alexandr Malusek, Ebba Helmrot, Michael Sandborg, J-E Grindborg and Gudrun Alm Carlsson, In-situ calibration of clinical built-in KAP meters with traceability to a primary standard using a reference KAP meter, 2014, Physics in Medicine and Biology, (59), 23, 71957210 .

http://dx.doi.org/10.1088/0031-9155/59/23/7195

Copyright: IOP Publishing: Hybrid Open Access http://www.iop.org/

Postprint available at: Linköping University Electronic Press http://urn.kb.se/resolve?urn=urn:nbn:se:liu:diva-112993 


\title{
In-situ calibration of clinical built-in KAP meters with traceability to a primary standard using a reference KAP meter
}

\author{
A Malusek ${ }^{1}$, E Helmrot ${ }^{2}$, M Sandborg ${ }^{1}$, J-E Grindborg ${ }^{3}$ and G Alm Carlsson ${ }^{1}$ \\ ${ }^{1}$ Radiation Physics, Department of Medical and Health Sciences, Center for Medical Image \\ Science and Visualization, Linköping University, Linköping, Sweden \\ ${ }^{2}$ Radiation Physics, Department of Medical and Health Sciences, Linköping University, \\ Linköping, Sweden \\ ${ }^{3}$ Swedish Radiation Protection Authority, Stockholm, Sweden
}




\begin{abstract}
The air kerma-area product (KAP) is used for settings of diagnostic reference levels. The International Atomic Energy Agency (IAEA) recommends that doses in diagnostic radiology (including the KAP values) be estimated with an accuracy of at least $\pm 7 \%(\mathrm{k}=2)$. Industry standards defined by the International Electrotechnical Commission (IEC) specify that the uncertainty of KAP meter measurements should be less than $\pm 25 \%(\mathrm{k}=2)$. Medical physicists willing to comply with the IAEA's recommendation need to apply correction factors to KAP values reported by $\mathrm{x}$-ray units. The aim of this work is to present and evaluate a calibration method for built-in KAP meters on clinical x-ray units. The method is based on (i) a tandem calibration method, which uses a reference KAP meter calibrated to measure the incident radiation, (ii) measurements using an energy-independent ionization chamber to correct for the energy dependence of the reference KAP meter, and (iii) Monte Carlo simulations of the beam quality correction factors that correct for differences between beam qualities at a standard laboratory and the clinic. The method was applied to the KAP meter in a Siemens Aristos FX plus unit. It was found that values reported by the built-in KAP meter differed from the more accurate values measured by the reference KAP meter by more than $25 \%$ for high tube voltages (more than 140 $\mathrm{kV})$ and heavily filtered beams $(0.3 \mathrm{~mm} \mathrm{Cu})$. Associated uncertainties were too high to claim that the IEC's limit of $25 \%$ was exceeded. Nevertheless the differences were high enough to justify the need for a more accurate calibration of built-in KAP meters.
\end{abstract}

\title{
1 Introduction
}

The air kerma-area product $P_{K A}$ is currently used for setting diagnostic reference levels (DRLs) in 11 European countries; see for instance the Dose Datamed 2 project (Bly et al 2011). The DRLs are determined as a percentile of the distribution of $P_{K A}$ values reported from all clinics in the region or country, or taken from other sources. The DRLs are expected not to be exceeded for standard procedures when good and normal practice regarding diagnostic and technical performance is applied (EC 1997). To make this procedure meaningful, reported $P_{K A}$ values need to be known with a sufficient degree of accuracy. Recent publications by ICRU (ICRU 2005) and IAEA (IAEA 2007) recommend that doses in diagnostic radiology be estimated with an accuracy of $\pm 7 \%$ (coverage factor 2 corresponding to a $95 \%$ confidence interval) including measurements of $P_{K A}$. For this to be achieved, careful calibration of clinically used kerma-area product (KAP) meters must be performed taking into account the strong energy dependence of commonly used commercially available KAP meters.

Today most clinical equipment for projection radiography and fluoroscopy has built-in KAP meters so that $P_{K A}$ values are automatically registered and displayed. The built-in system may alternatively be based on software that calculates $P_{K A}$ from information on the settings of collimators, tube voltage $(\mathrm{kV})$ and tube charge $(\mathrm{mAs})$. The method of calibration used by the manufacturer is often based on a measurement at one beam quality only; the details are not easily available. Industry standards (IEC 2000) define that the uncertainty of KAP meter measurement should be less than $\pm 25 \%$ ( $k=2$ ). For manufacturers this high limit means the large energy dependence of KAP meters can be neglected. For radiation safety authorities, however, it adds uncertainty in reported $P_{K A}$ data from clinics and makes data analysis more difficult.

To check the calibration coefficients and determine the energy dependence of the built-in KAP meters, physicists need to have access to a measurement system that registers the $P_{K A}$ incident on the patient, i.e., transmitted through the built-in KAP meter. For this, a KAP meter (here called 
reference KAP meter) may be used that, in principle, registers $P_{\mathrm{KA}}$ according to the definition. This method of performing $P_{K A}$ measurements (Toroi et al 2008) has to some extent replaced the earlier method used in the NRPB protocol (NRPB 1992) that requires measurement of the beam area using film as detector.

The aim of this work is to develop a method improving the accuracy of clinical KAP meter measurements based on the use of a reference KAP meter; the new calibration coefficients should be traceable to a primary standard. A reference KAP meter calibrated at the Secondary Standards Dosimetry Laboratory (SSDL) in Stockholm (the Swedish Radiation Safety Authority (SSM)) will be used and the transfer of the calibration coefficient from the SSDL to the clinical KAP meter will be determined via computer simulations.

\section{Materials and methods}

Monte Carlo simulations of the response of a typical KAP meter as a function of photon energy will be used to derive calibration coefficients for energy spectra of photons at the SSDL and in the clinics, respectively. While the absolute sensitivity (the calibration coefficient $N_{P_{K A}, Q}$ ) of individual KAP meters of nominally the same make may vary by as much as almost $40 \%$ (see Section 3.1) beam quality correction factors $k_{Q, Q_{0}}\left(Q=\right.$ user beam quality, $Q_{0}=$ reference beam quality) being the quotient of the calibration coefficients at the two beam qualities, are only little influenced by variations in absolute sensitivity. Simulations can thus not be used to predict absolute sensitivity, which has to be handled via calibration of each KAP meter at the SSDL.

Simulation of the response of a commercially available KAP meter (Vacutec 70157) is described in 2.1.1, derivation of energy spectra in 2.1.2, calculation of the calibration coefficient and beam quality correction factor in 2.1.3. Experiments performed to verify the simulations at the calibration laboratory and in the clinic are described in 2.2.

\section{$2.1 \quad$ Calculations}

\subsubsection{Monte Carlo simulation of KAP meter response}

Energy imparted to air cavities of the Vacutec 70157 KAP meter was simulated using the penmain code of the Penelope 2011 package (Salvat and Fernández-Varea 2009). A schematic view of the irradiation setup modeling the calibration geometry at the SSDL in Stockholm, Sweden (SSM) and the model of the KAP meter are shown in figure 1. The entrance surface of the KAP meter was positioned $1000.0 \mathrm{~mm}$ from the focal spot. A tungsten collimator $3 \mathrm{~mm}$ thick was positioned 50 $\mathrm{mm}$ from the KAP meter. The collimator had a rectangular opening of $47.45 \mathrm{~mm} \times 47.45 \mathrm{~mm}$, which formed a beam with the field size of $50.0 \mathrm{~mm} \times 50.0 \mathrm{~mm}$ at the entrance surface of the KAP meter. The model of the KAP meter consisted of a PMMA body with dimensions of $164 \mathrm{~mm} \times 164$ $\mathrm{mm} \times 15.9 \mathrm{~mm}$. The size of each of the two air cavities was $147 \mathrm{~mm} \times 147 \mathrm{~mm} \times 5.9 \mathrm{~mm}$. Thicknesses of the outer and inner electrodes were 1.5 and $1.0 \mathrm{~mm}$, respectively. Surfaces of the inner and outer electrodes facing the air cavities were covered with a conductive layer of 10,15, or $20 \mathrm{~nm}$ of $\mathrm{In}_{2} \mathrm{O}_{3}$. 

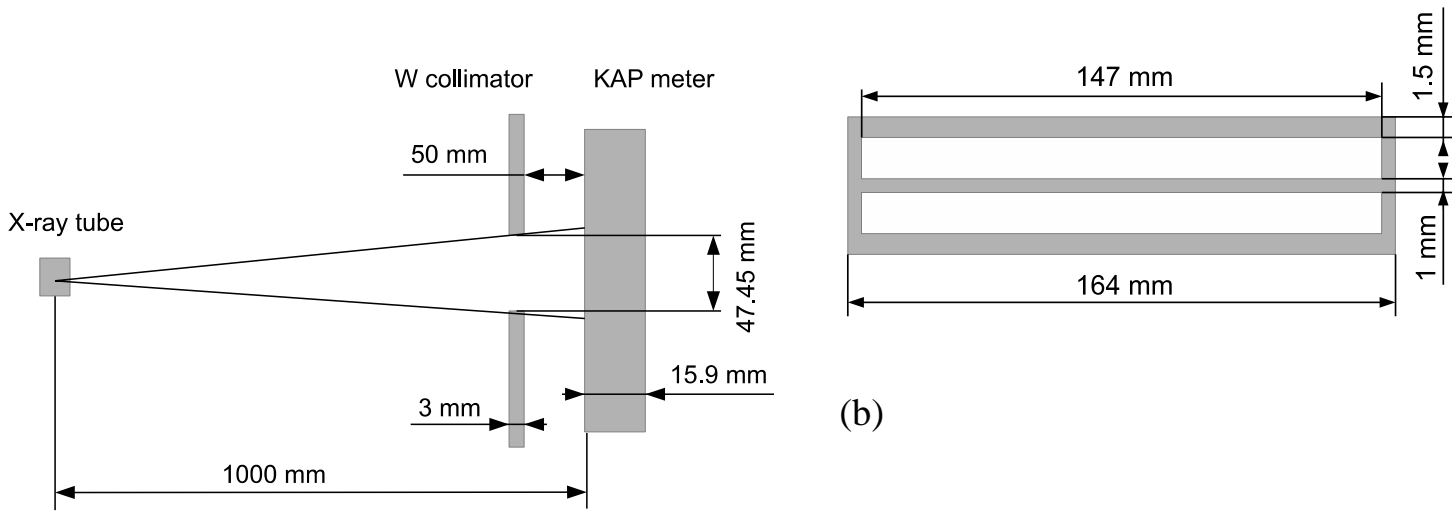

(b)

(a)

Figure 1: (a) Schematic view of the irradiation setup. (b) Schematic view of the KAP meter. These geometries (not drawn to scale) were used for both the Monte Carlo simulations of detector response and calibration measurements.

Monoenergetic photons with energy $E_{i}$ were emitted from a point source to a circular cone with the aperture $\alpha=5.73^{\circ}$ measured from the beam axis; the field covered an area larger than the collimator opening. Energies $E_{i}$ covered the range from 4 to $150 \mathrm{keV}$, see the markers in figure 2. A separate simulation was executed for each of the energies $E_{i}$.

Energy, $\varepsilon^{\prime}$, imparted to air cavities of the KAP meter per one emitted photon was scored by the penmain code. This quantity was normalized per 1 photon emitted to the solid angle of $4 \pi$ using $\varepsilon=0.5(1-\cos \alpha) \varepsilon^{\prime}$. The function $\varepsilon(E)$ is shown in figure 2 .

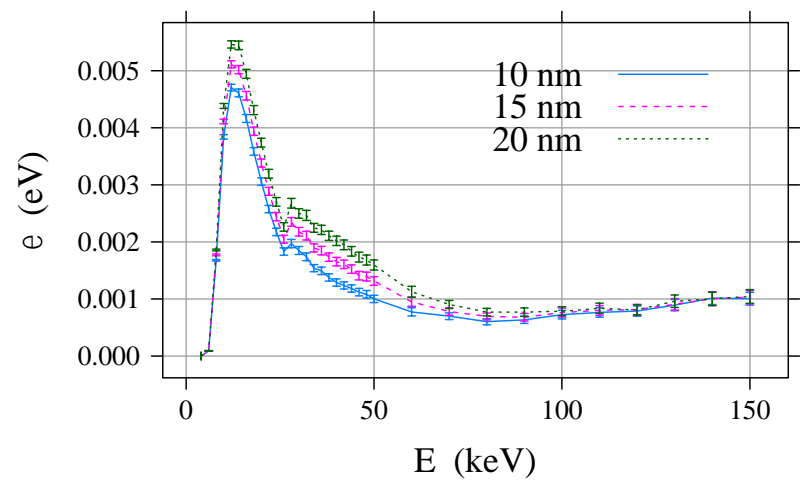

Figure 2: Energy $\varepsilon(E)$ imparted to the air cavity as a function of photon energy $E$ for conductive layer thicknesses of 10, 15 and $20 \mathrm{~nm}$. Error bars show expanded statistical uncertainty $(k=3)$.

Figure 2 shows that the response of the chamber depends on the thickness of the In-layer, particularly at photon energies in the range $30-50 \mathrm{keV}$. The discontinuity in the curve at $29.7 \mathrm{keV}$ is due to the K-edge of In.

For a polyenergetic beam, a point source emitting $s_{\Omega, E}(E) d E d \Omega$ photons with energy in the interval $(E, E+d E)$ to the solid angle $d \Omega$ was approximated with a source having discrete energy spectrum, $w\left(E_{i}\right)$, sampled at equidistant energies $E_{i}, 1 \leq i \leq N$, where $N$ is the number of bins. 
The number of photons in a bin with energy $E_{i}$ emitted from the source to the solid angle of $4 \pi \mathrm{sr}$ is then $w\left(E_{i}\right) \cong 4 \pi s_{\Omega, E}\left(E_{i}\right) \Delta E$, where $\Delta E$ is the bin width. Energy spectra $w\left(E_{i}\right)$ were obtained from the SpekCalc program (Poludniowski et al 2009), see Section 2.1.2. They were normalized so that $\sum_{i=1}^{N} w\left(E_{i}\right)=1$ corresponding to 1 photon emitted to the solid angle of $4 \pi \mathrm{sr}$.

The energy, $\varepsilon_{s}$, imparted to air cavities per one photon emitted to the solid angle of $4 \pi$ sr was calculated by approximating the integral over all photon energies with a sum over all energy spectrum bins as

$$
\varepsilon_{s}=\int_{0}^{\infty} 4 \pi s_{\Omega, E}(E) \varepsilon(E) d E \cong \sum_{i=1}^{N} 4 \pi s_{\Omega, E}\left(E_{i}\right) \Delta E \varepsilon\left(E_{i}\right)=\sum_{i=1}^{N} w\left(E_{i}\right) \varepsilon\left(E_{i}\right),
$$

where $w\left(E_{i}\right)$ is the energy spectrum and $\varepsilon\left(E_{i}\right)$ is the energy imparted to KAP meter air cavities by monoenergetic photons. Values of $\varepsilon\left(E_{i}\right)$ at energy $E_{i}$ were obtained by linear interpolation between values $\varepsilon\left(E_{i}^{\prime}\right)$ and $\varepsilon\left(E_{i}^{\prime \prime}\right)$ calculated with PENELOPE at energies $E_{i}^{\prime}$ and $E_{i}^{\prime \prime}$, respectively. These energies were chosen so that the interval $\left(E_{i}^{\prime}, E_{i}^{\prime \prime}\right)$ was the smallest interval containing the energy $E_{i}$. The collected charge was calculated as

$$
Q=\frac{e}{W} \varepsilon_{s}
$$

where $\mathrm{W} / \mathrm{e}=33.97 \mathrm{JC}^{-1}$ is the mean energy required for the creation of an electron-ion pair (Boutillon and Perroche-Roux 1987). Free-in-air air collision kerma, $K_{c, \text { air }}$, was calculated as

$$
K_{c, a i r}=\int_{0}^{\infty} E \Phi_{E}(E) \frac{\mu_{e n}(E)}{\rho_{\text {air }}} d E \cong \sum_{i=1}^{N} E_{i} \frac{w\left(E_{i}\right)}{4 \pi d} e^{-\mu\left(E_{i}\right) d} \frac{\mu_{e n}\left(E_{i}\right)}{\rho_{\text {air }}},
$$

where $\mu_{e n}(E) / \rho_{\text {air }}$ is the mass energy absorption coefficient for dry air at sea level, $\mu(E)$ is the linear attenuation coefficient for air at photon energy $E, d=1 \mathrm{~m}$ is the source-detector distance, and $w\left(E_{i}\right)$ is the energy spectrum. In (3), the distribution of fluence $\Phi_{E}(E)$ of primary photons with respect to energy at the distance of $1 \mathrm{~m}$ from the point source was estimated as

$$
\Phi_{E}(E)=\frac{s_{\Omega, \mathrm{E}}(E)}{d^{2}} \exp [-\mu(E) d]
$$

Values of the linear attenuation coefficient for air were calculated from photon cross sections in the EPDL97 library (Cullen 1997). Values of the mass energy absorption coefficient for air were taken from (Hubbell and Seltzer 2004).

Uncertainties associated with $\varepsilon_{s}$ were estimated using the Guide to the expression of Uncertainty in Measurement (GUM) also known as GUM 1995 with minor corrections (JCGM, 2008), see the Appendix. Only uncertainties owing to the statistical nature of the Monte Carlo simulation were considered. Uncertainties associated with the spectra were assumed to affect the calibration coefficient of the KAP meter (section 2.1.3) only little and were neglected. Though random variations in the spectrum shapes would notably affect both $K_{c, \text { air }}$ and $Q$ (see equations (1) and (2)), the ratio $K_{c, \text { air }} / Q$ would be affected to a much smaller degree owing to a positive covariance between $K_{c, \text { air }}$ and $Q$ (cf. section 4.2). 


\subsubsection{Derivation of energy spectra}

Calibration spectra with RQR and RQA beam qualities (IAEA 2007) were calculated using SpekCalc for the tube voltages and added filtrations shown in table 1. The x-ray tube at the SSDL in Stockholm had a tungsten anode, inherent filtration of $3 \mathrm{~mm}$ of Be and anode angle of $20^{\circ}$. Quality of the calculated energy spectra was checked by comparing calculated half-value layer (HVL) with HVL measured in narrow beam geometry according to (IAEA 2007). To match the calculated HVL with the measured HVL an extra filter of tungsten (thickness given in table 1) was added to the calculated beams. The need for the extra filtration by tungsten was most likely caused by the roughness of the anode, which increases with the x-ray tube wear (Nowotny and Meghzifene 2002). A deposit of tungsten on the exit window of the tube may also play a role (Stears et al 1986, Nagel 1988), although corresponding values should be significantly lower. The extra filtration by tungsten was not needed for the RQA beam qualities, where the heavy filtration by aluminum decreased the effect of anode roughness and other construction materials in the x-ray tube.

Table 1: Tube voltage $U$, total thickness of aluminum $t_{\mathrm{Al}}$, and the measured half value layer of aluminum $\mathrm{HVL}_{\mathrm{m}}$ for the RQR and RQA beam qualities $Q$ at SSM. Corresponding half value layer of aluminum calculated using SpekCalc $\mathrm{HVL}_{\mathrm{c}}$, the relative difference $\delta=\left(H V L_{c}-\right.$ $\left.H V L_{m}\right) / H V L_{m}$, and the thickness of tungsten $t_{\mathrm{W}}$ that results in the same measured and calculated HVLs are also listed.

\begin{tabular}{lrrrrrr}
\hline$Q$ & $\begin{array}{r}U \\
(\mathrm{kV})\end{array}$ & $\begin{array}{r}t_{\mathrm{Al}} \\
(\mathrm{mm})\end{array}$ & $\begin{array}{r}\mathrm{HVL}_{\mathrm{m}} \\
(\mathrm{mm})\end{array}$ & $\begin{array}{r}\mathrm{HVL}_{\mathrm{c}} \\
(\mathrm{mm})\end{array}$ & $\begin{array}{r}\delta \\
(\%)\end{array}$ & $\begin{array}{r}t_{\mathrm{W}} \\
(\mu \mathrm{m})\end{array}$ \\
\hline RQR 2 & 40 & 2.51 & 1.44 & 1.38 & -4.2 & 1.6 \\
RQR 3 & 50 & 2.51 & 1.80 & 1.72 & -4.4 & 1.6 \\
RQR 4 & 60 & 2.77 & 2.23 & 2.14 & -4.0 & 1.6 \\
RQR 5 & 70 & 2.77 & 2.54 & 2.43 & -4.3 & 1.6 \\
RQR 6 & 80 & 3.04 & 3.04 & 2.89 & -4.9 & 1.8 \\
RQR 7 & 90 & 3.04 & 3.40 & 3.24 & -4.7 & 1.8 \\
RQR 8 & 100 & 3.50 & 4.07 & 3.90 & -4.2 & 1.9 \\
RQR 9 & 120 & 3.85 & 5.06 & 4.90 & -3.2 & 1.7 \\
RQR 10 & 150 & 4.50 & 6.64 & 6.49 & -2.3 & 1.7 \\
& & & & & & \\
RQA 2 & 40 & 6.55 & 2.20 & 2.18 & -0.9 & \\
RQA 3 & 50 & 13.51 & 3.85 & 3.86 & 0.3 & \\
RQA 4 & 60 & 18.76 & 5.38 & 5.34 & -0.7 & \\
RQA 5 & 70 & 23.79 & 6.79 & 6.76 & -0.4 & \\
RQA 6 & 80 & 29.10 & 8.17 & 8.16 & -0.1 & \\
RQA 7 & 90 & 33.08 & 9.27 & 9.25 & -0.2 & \\
RQA 8 & 100 & 37.56 & 10.20 & 10.21 & 0.1 & \\
RQA 9 & 120 & 43.86 & 11.71 & 11.69 & -0.2 & \\
RQA 10 & 150 & 49.56 & 13.39 & 13.39 & 0.0 & \\
\hline
\end{tabular}

Clinical energy spectra for the Siemens Axiom Aristos FX unit were calculated using SpekCalc for the tube voltages and beam filtrations shown in table 2 . Tube voltages $70-80 \mathrm{kV}$ are representative of abdomen, pelvis and lumbar spine examinations (added $\mathrm{Cu}$ filtrations $0.1-0.3 \mathrm{~mm}$ ), tube voltages $140-150 \mathrm{kV}$ with added $\mathrm{Cu}$ filters $0.1-0.3 \mathrm{~mm}$ are representative of chest examinations. Anode material was set to tungsten, anode angle was 12 degrees, and 
total filtration of $3 \mathrm{~mm}$ of $\mathrm{Al}$ was used. Anode material of a clinical $\mathrm{x}$-ray tube is typically tungsten with additions of rhenium or molybdenum. Anode angles are between 8 and 12 degrees, but other values are also possible. Total filtration of the $\mathrm{x}$-ray tube assembly is typically about $2.5-3.5 \mathrm{~mm}$ Al.

The quality of the calculated energy spectra were tested by comparing HVL calculated by the SpekCalc program and measured HVL for the clinical beam qualities used. Results shown in Figure 3 indicate good agreement between calculated and real energy spectra; differences between calculated and measured values of HVL relative to measured values of HVL were less than 3\%. The multi-purpose detector with the Barracuda instrument (RTI Electronics Inc.) was used for the HVL measurements; according to manufacturer's specifications, relative expanded uncertainty $(\mathrm{k}=2)$ in determining HVL values was $10 \%$ or $0.2 \mathrm{~mm}$ of $\mathrm{Al}$, see (RTI 2005) for more details.

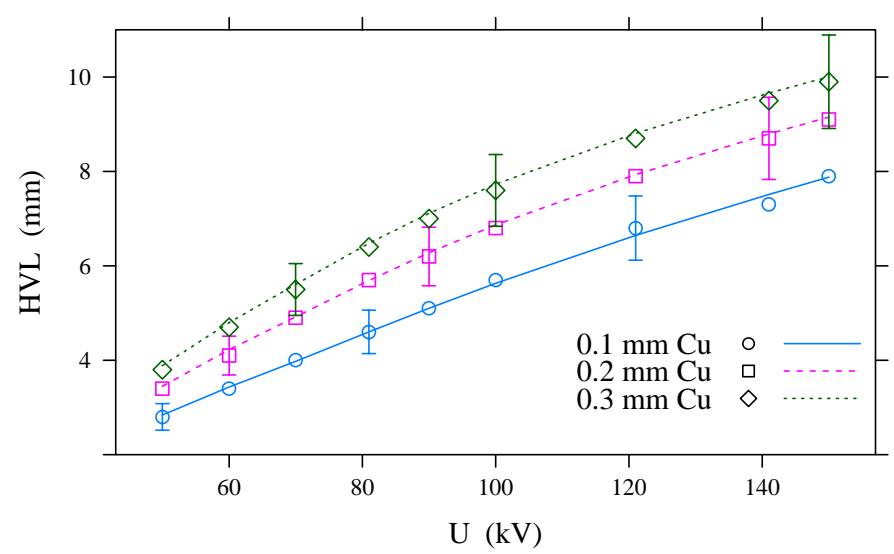

Figure 3: Calculated (lines) and measured (markers) HVL of aluminum for beam filtrations of $0.1,0.2$ and $0.3 \mathrm{~mm} \mathrm{Cu}$ of the (Siemens Axiom Aristos FX plus) $\mathrm{x}$-ray stand as a function of the tube voltage $U$. Error bars showing expanded uncertainty $(\mathrm{k}=2)$ are plotted at every third point to avoid overlapping.

\subsubsection{Derivation of beam quality correction factors}

The calibration coefficient for the beam of interest (quality $Q$ ) is given by

$$
N_{P_{K A, Q}}=\frac{P_{K A, Q}}{M_{Q}},
$$

where $P_{K A, Q}$ is the air kerma-area product and $M_{Q}$ is the signal from the KAP meter. The same definition applies to the calibration beam at reference beam quality $Q_{0}$.

The beam quality correction factor, $k_{Q, Q_{0}}$, for a KAP meter used at beam quality $Q$ and calibrated using the reference beam $Q_{0}$ is given by

$$
k_{Q, Q_{0}}=\frac{N_{P_{K A, Q}}}{N_{P_{K A, Q_{0}}}} .
$$

The calculated calibration coefficients were obtained using

$$
P_{K A}=K_{c, a i r} A
$$

with $K_{c, \text { air }}$ from equation (3), the area $A$ from the calibration geometry shown in Figure 1(a) and the signal $M$ equal to the charge $Q$ in equation (2). 


\subsubsection{Transfer of the calibration coefficient at the calibration laboratory to the built-in KAP meter}

Let $M_{Q}^{\text {int }}$ be the charge collected by the clinical (built-in) KAP meter and $P_{K A, Q}^{t}$ be the true value that the clinical KAP meter estimates. The true calibration coefficient of the clinical KAP meter is then

$$
N_{P_{K A}, Q}^{i n t, t}=\frac{P_{K A, Q}^{t}}{M_{Q}^{i n t}} .
$$

The calibration coefficient $N_{P_{K A}, Q}^{\text {int }}$ used by the machine and the charge $M_{Q}^{\text {int }}$ collected by the builtin KAP meter are typically not available to the hospital physicist. It is, however, possible to calculate the correction factor, here denoted $\kappa$, that converts the calibration coefficient of the builtin KAP meter $N_{P_{K A}, Q}^{\text {int }}$ to the true calibration coefficient $N_{P_{K A}, Q}^{\text {int, } t}$ as

$$
\kappa \equiv \frac{N_{P_{K A}, Q}^{\text {int },}}{N_{P_{K A}, Q}^{\text {int }}}=\frac{N_{P_{K A}, Q}^{\text {int }, t} M_{Q}^{\text {int }}}{N_{P_{K A}, Q}^{\text {int }} M_{Q}^{\text {int }}}=\frac{P_{K A, Q}^{t}}{P_{K A, Q}^{\text {int }}},
$$

where $P_{K A, Q}^{t}$ and $P_{K A, Q}^{i n t}$ are the true value and the (in general biased) reading, respectively, of the built-in KAP meter.

The true value $P_{K A, Q}^{t}$ (determined by the reference KAP meter) can formally be written as

$$
P_{K A, Q}^{t}=N_{P_{K A}, Q}^{r e f} M_{Q}^{r e f}=\frac{N_{P_{K A}, Q}^{r e f}}{N_{P_{K A}, Q_{1}}^{r e f}} \frac{N_{P_{K A}, Q_{1}}^{r e f}}{N_{P_{K A}, Q_{0}}^{r e f}} N_{P_{K A}, Q_{0}}^{r e f} M_{Q}^{r e f}=k_{Q, Q_{1}}^{r e f} k_{Q_{1}, Q_{0}}^{r e f} N_{P_{K A}, Q_{0}}^{r e f} M_{Q}^{r e f},
$$

where $N_{P_{K A}, Q}^{r e f}, N_{P_{K A}, Q_{0}}^{r e f}$ and $N_{P_{K A}, Q_{1}}^{r e f}$ are calibration coefficients of the reference KAP meter for beam qualities $Q, Q_{0}$ and $Q_{1}$, respectively, $M_{Q}^{r e f}$ is the signal of the reference KAP meter for the beam quality $Q$, and $k_{Q, Q_{1}}^{r e f}$ and $k_{Q_{1}, Q_{0}}^{r e f}$ are the corresponding beam quality correction factors. The beam quality correction factor $k_{Q_{1}, Q_{0}}^{r e f}$ converts the calibration coefficient from the reference beam quality $Q_{0}$ used at the calibration laboratory (e.g., RQR 5) to a reference beam quality $Q_{l}$ used at the clinics and represents the transfer of the calibration coefficient from the calibration laboratory to the calibration at the clinic. The beam quality correction factor $k_{Q, Q_{1}}^{r e f}$ converts the calibration coefficient from the reference beam quality $Q_{1}$ used at the clinic to the beam quality $Q$ used for the examination.

The beam quality correction factor $k_{Q_{1}, Q_{0}}^{r e f}$ cannot be experimentally determined but has to be derived using model based simulations. The beam quality correction factor $k_{Q, Q_{1}}^{r e f}$ can be simulated but also determined experimentally using an energy independent ionization chamber as described below in 2.2.2.

\section{Experiments}

\subsubsection{Experiments at the calibration laboratory}

The KAP meter (Vacutec 70157) simulated in 2.1.1 was calibrated at the SSDL using the geometry shown in Figure 1(a) and the set of standard beam qualities RQR and RQA in table 1. Results were 
used to determine which of the geometry models (thicknesses of the indium layer), figure 1(b), of the KAP meter best fitted the measured KAP meter response.

\subsubsection{Experiments in the clinic}

Measurements were performed at a laboratory typical of examinations where DRLs are based on the quantity $P_{K A}$. This section describes (i) the x-ray equipment and imaging parameters, (ii) measurements of the energy dependence of the reference KAP meter, and (iii) derivation of experimentally determined relative errors of calibration coefficients of a clinical built-in KAP meter.

The Siemens Axiom Aristos FX Plus unit contained a Siemens x-ray tube (OPTI 150/30/50 HC100 , tungsten-rhenium coated anode, anode angle of 12 degrees) and a built-in KAP meter (KERMAX, Scanditronix Wellhöfer $\mathrm{GmbH}$ ). Tube voltages $50-150 \mathrm{kV}$ were chosen for the measurements and added $\mathrm{Cu}$-filtrations of $0.0,0.1,0.2$ and $0.3 \mathrm{~mm}$.

The calibrated reference KAP meter should be used to measure the air kerma-area product of the beam incident on the patient in the absence of the KAP meter. This puts requirements on how it is positioned in the beam. It must be positioned sufficiently close to the collimator so that the entire primary beam is covered. On the other hand, it must be positioned at a sufficient distance from the collimator housing and the built-in KAP meter (and any other material in the beam in front of the reference KAP meter) to avoid influencing the signal from the built-in KAP meter (through backscattered radiation) or registering very oblique stray radiation from the housing and any material in the beam between the housing and the reference KAP meter. Radiation transmitted at large angles to the central axis of the beam will not reach the patient and should thus not be registered. Work by Toroi et al (2008) and Malusek and Alm Carlsson (2011) show that a distance of 20-30 cm between the reference KAP meter and the collimator housing is sufficient to avoid registration of such scattered photons. The geometry used in the clinical measurements is shown in figure 4.

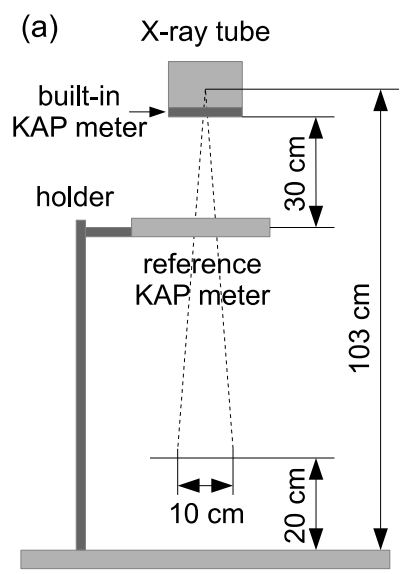

patient table

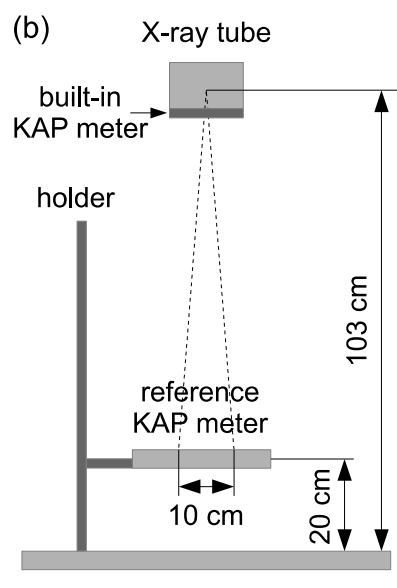

patient table

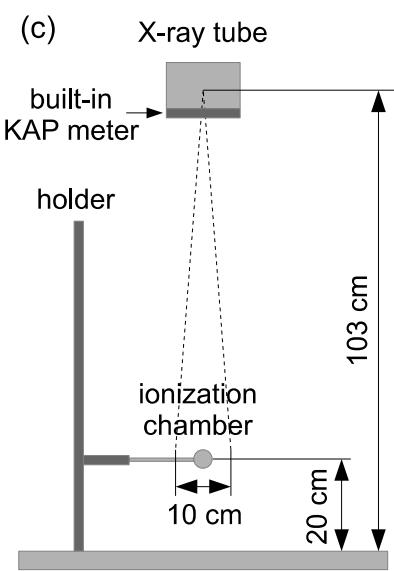

patient table

Figure 4: (a) Calibration of the built-in KAP meter of the Siemens Aristos FX plus unit. The reference KAP meter was positioned about $30 \mathrm{~cm}$ from the built-in KAP meter. The field size was $10 \mathrm{~cm} \times 10 \mathrm{~cm}$ at the distance of $20 \mathrm{~cm}$ from the patient table. (b, c) Energy dependence of the reference KAP meter was measured using an ionization chamber with air equivalent walls; the built-in KAP meter was used as the monitor chamber in the substitution method described in (IAEA 2007). 
The $P_{K A}$ value displayed by the built-in KAP meter was registered at each measurement with the reference KAP meter and with the ionization chamber.

\subsubsection{Experimental verification of the simulated calibration coefficients for the clinical beams}

Using an energy independent ionization chamber positioned on the central axis of the beam at a nominal field size, $A_{\text {nom }}$, the energy dependence of the reference KAP meter can be evaluated and compared to the calculated values. The field size (collimator setting) was the same as that used in the geometry with the reference KAP meter and the ionization chamber was placed at the same distance as the reference KAP meter from the focal spot. A position $20 \mathrm{~cm}$ above the patient table was used according to (IAEA 2007) recommendations to avoid the influence of backscatter. A spherical ionization chamber (Shonka Exradin) with low energy dependence was used; maximum relative difference between its calibration coefficients obtained at SSM for RQR 2-10, RQA 2-10 and RQT $8-10$ beam qualities was $2.4 \%$.

The beam quality correction factor $k_{Q, Q_{1}}$ can be calculated similar to equation (6) where the calibration coefficients for beam qualities $Q$ and $Q_{1}$ are estimated using

$$
\begin{aligned}
& N_{P_{K A, Q}} \equiv \frac{P_{K A, Q}}{M_{Q}}=\frac{K_{\text {air }, \text { center }, Q} A_{\text {nom }, Q}}{M_{Q}} \\
& N_{P_{K A, Q_{1}}} \equiv \frac{P_{K A, Q_{1}}}{M_{Q_{1}}}=\frac{K_{\text {air }, \text { center }, Q_{1}} A_{\text {nom }, Q_{1}}}{M_{Q_{1}}} .
\end{aligned}
$$

In (11) $M_{Q}$ is the reading of the reference KAP meter and $K_{\text {air,center, } Q}$ is the air kerma measured by the ionization chamber at the center of the field with the nominal size of $A_{n o m, Q}$ for the beam quality $Q$. Similar notation is used for the beam quality $Q_{1}$ in (12). The field sizes (the collimator settings) are the same for beam qualities $Q$ and $Q_{1}$ and (6) simplifies to

$$
k_{Q, Q_{1}} \equiv \frac{N_{P_{K A, Q}}}{N_{P_{K A, Q_{1}}}}=\frac{K_{c, \text { air }, Q}}{K_{c, \text { air }, Q_{1}}} \frac{M_{Q_{1}}}{M_{Q}} .
$$

2.3 Error in calibration coefficients used by the built-in KAP meter

Relative error, $\delta$, in the calibration coefficient of the built-in KAP meter was calculated as

$$
\delta \equiv \frac{N_{P_{K A}, Q}^{i n t}-N_{P_{K A}, Q}^{i n t, t}}{N_{P_{K A}, Q}^{i n t, t}}=\frac{1}{\kappa}-1=\frac{P_{K A, Q}^{i n t}}{P_{K A, Q}^{t}}-1,
$$

where $N_{P_{K A}, Q}^{\text {int, }}$ and $N_{P_{K A}, Q}^{\text {int }}$ are the true and used, respectively, calibration coefficients of the built-in KAP meter, $\kappa$ is defined in equation (9), and $P_{K A, Q}^{t}$ and $P_{K A, Q}^{i n t}$ are the true value and the reading, respectively, of the built-in KAP meter. The true value $P_{K A, Q}^{t}$ was estimated as the value of $P_{K A, Q}^{\text {ref }}$ provided by the reference KAP meter, see equation (10). The beam quality $Q_{1}$ in (12) corresponded to $70 \mathrm{kV}$ and $0.0 \mathrm{~mm} \mathrm{Cu}$. Beam qualities $Q$ were given by the added filtration of $0.0,0.1$ and 0.3 $\mathrm{mm} \mathrm{Cu}$ and tube voltages $50 \mathrm{kV}-150 \mathrm{kV}$. 


\section{Results}

Simulated calibration coefficients and calibration coefficients derived from measurements in the calibration beams are shown in figure 5, where labels KAP 1,2 and 3 denote data from three different chambers of the same model (Vacutec 70157); data from RQA beams were not available for KAP 3. Measured calibration coefficients were determined from equation (5); associated relative uncertainties of $2.4 \%(\mathrm{k}=2)$ were provided by SSM. Large variations in measured values of $N_{P_{K A}}$ among different KAP meters of the same model are most likely caused by different thickness of their conductive layers. Figure 5 indicates e.g. that the thickness was slightly less than $15 \mathrm{~nm}$ for the KAP meter 1.
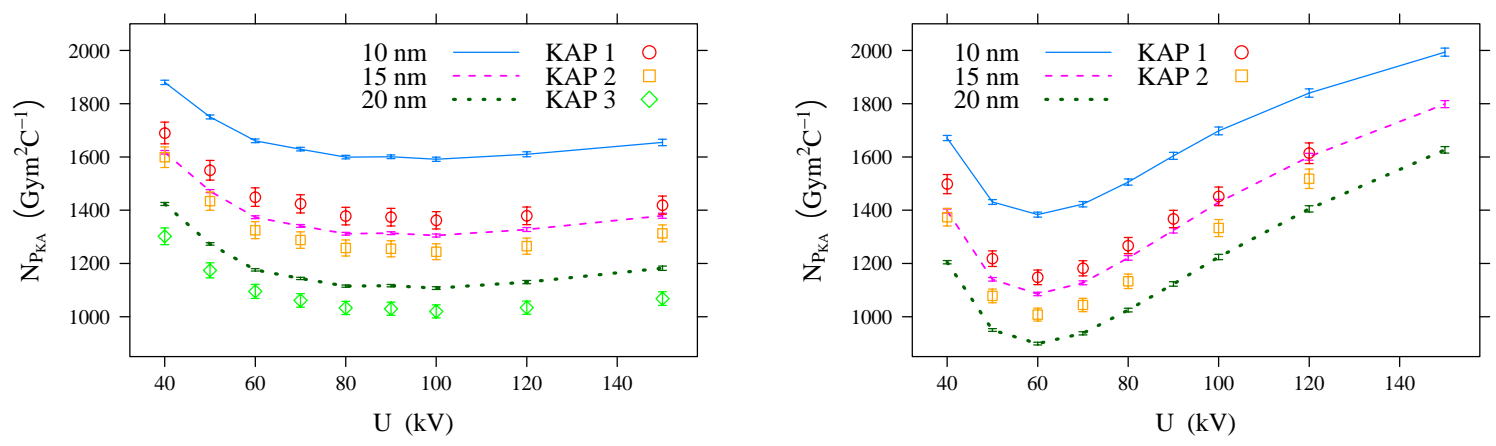

Figure 5: Calibration coefficients $N_{P_{K A}}$ as functions of tube voltage $U$ for RQR (left) and RQA (right) beam qualities. Calculated values for conductive layer thicknesses 10, 15 and $20 \mathrm{~nm}$ are plotted with lines. Measured values for KAP meters 1, 2 and 3 are plotted with markers. Error bars show uncertainties for measured $(\mathrm{k}=2)$ and simulated $(\mathrm{k}=3)$ values.

3.2 Measured and calculated beam quality correction factors for the calibration beams The RQR 5 beam quality was taken as the reference beam quality $Q_{0}$ as it is often used for the calibration of clinical KAP meters. Measured values of calibration coefficients relative to the calibration coefficient for RQR 5 are shown in figure 6 for the three KAP meters 1, 2 and 3 of the Vacutec 70157 model. Measured values have a relative uncertainty of $3.4 \%(\mathrm{k}=2)$. Though their calibration coefficients noticeably varied for a given tube voltage (cf. figure 5), the variation in the beam quality correction factor $k_{Q, Q_{0}}$ among the KAP meters was mostly within $3 \%$, the largest relative differences of $4.6 \%$ and $4.0 \%$ were for RQR 2 and RQA 9. Calculated values of $k_{Q, Q_{0}}$ fit the data points well. 



Figure 6: Beam quality correction factors $k_{Q, Q_{0}}=N_{P_{K A}, Q} / N_{P_{K A}, Q_{0}}$ as functions of tube voltage $U$ for RQR (left) and RQA (right) beam qualities. Calculated values for conductive layer thicknesses 10,15 and $20 \mathrm{~nm}$ are plotted with lines. Measured values for KAP meters 1,2 and (in case of RQR) 3 are plotted with markers. RQR 5 was used as the reference beam quality $Q_{0}$. Error bars show uncertainties $(\mathrm{k}=2)$ for measured values. Uncertainties of simulated values were less than $1 \%$ $(\mathrm{k}=3)$. They are not plotted to ease readability.

\subsection{Measured and calculated beam quality correction factors for the clinical beams}

Measured and calculated beam quality correction factors $k_{Q, Q_{1}}$ for the reference KAP meter and the Siemens Axiom Aristos FX x-ray stand are shown in figure 7, the measured values are also shown in table 2. The reference beam quality $Q_{1}$ was set to $70 \mathrm{kV}$ and no extra filtration of $\mathrm{Cu}$. Measured values were determined from equation (13), associated relative uncertainties of $3 \%(\mathrm{k}=2)$ were calculated using GUM 1995 by assuming that relative uncertainties $(\mathrm{k}=2)$ for $K_{c, \text { air }}$ and $M_{Q}$ were $2 \%$ and $1 \%$, respectively. There was a relatively good agreement between measured and calculated data for the conductive layer thickness of $15 \mathrm{~nm}$; differences between calculated and measured values relative to measured values were less than $3.2 \%$ and $5.4 \%$ for the RQR and RQA beam qualities, respectively. The real thickness of the conductive layer is most likely slightly less than 15 $\mathrm{nm}$. This finding was consistent with measurements at the calibration laboratory (figures 5,6). Figure 7 also shows that added filtration may significantly change the value of the calibration coefficient compared to the calibration coefficient for a spectrum filtered by the inherent filtration of approximately $3 \mathrm{~mm} \mathrm{Al}$ only. 


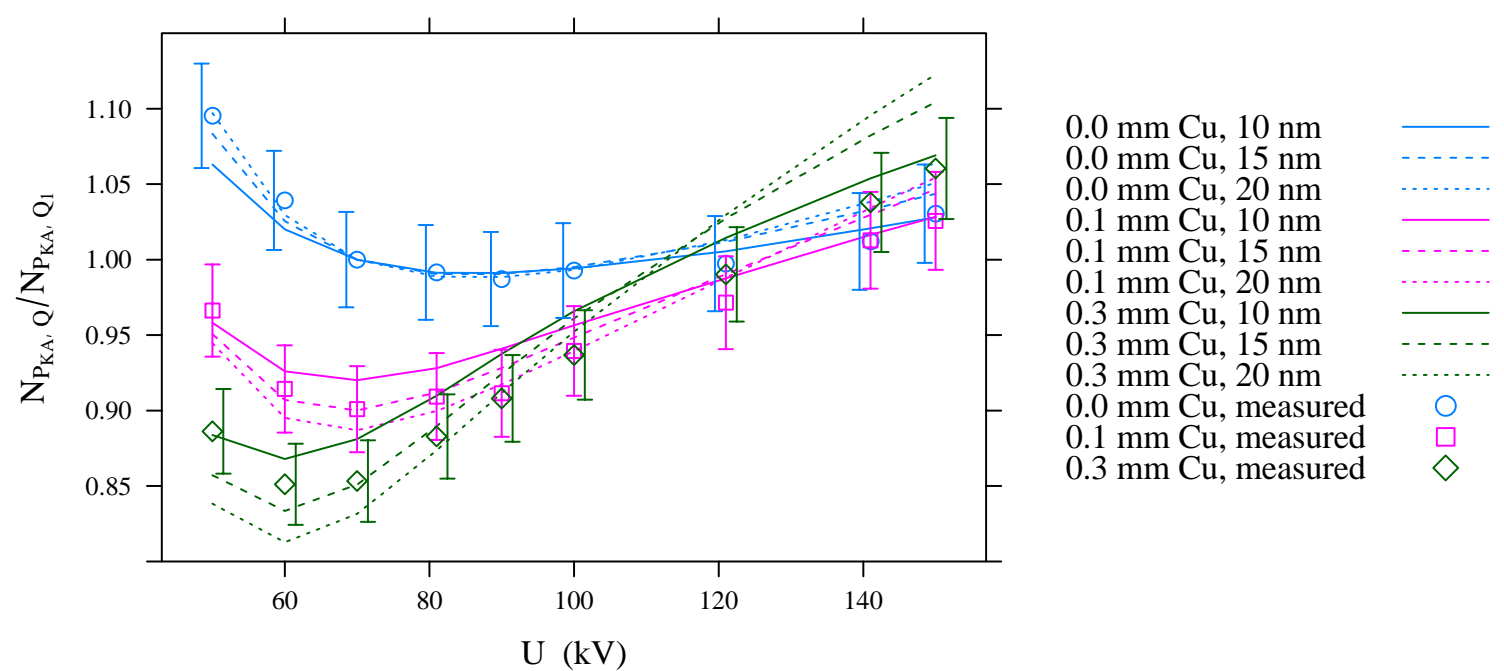

Figure 7: Calculated (lines) and measured (markers) beam quality correction factors for the reference KAP meter at beam filtrations of $0.0,0.1$ and $0.3 \mathrm{~mm} \mathrm{Cu}$ of the Axiom Aristos x-ray stand. Calculations were done for conductive layer thicknesses of 10, 15 and $20 \mathrm{~nm}$. Error bars showing uncertainties $(\mathrm{k}=2)$ are adjusted in horizontal direction to avoid overlapping.

Table 2: Experimentally determined beam quality correction factors $k_{Q, Q_{1}}$ for the (reference) Vacutec 70157 KAP meter and Siemens Aristos FX plus x-ray stand as a function of the tube voltage $U$ for additional filtrations of $0.0,0.1$ and $0.3 \mathrm{~mm} \mathrm{Cu}$. The reference beam quality $Q_{1}$ was set to $70 \mathrm{kV}$ and $0.0 \mathrm{~mm} \mathrm{Cu}$.

\begin{tabular}{llll}
\hline $\mathrm{U}(\mathrm{kV})$ & $0.0 \mathrm{~mm} \mathrm{Cu}$ & $0.1 \mathrm{~mm} \mathrm{Cu}$ & $0.3 \mathrm{~mm} \mathrm{Cu}$ \\
\hline 50 & $1.10 \pm 0.04$ & $0.97 \pm 0.03$ & $0.89 \pm 0.03$ \\
60 & $1.04 \pm 0.03$ & $0.91 \pm 0.03$ & $0.85 \pm 0.03$ \\
70 & $1.00 \pm 0.03$ & $0.90 \pm 0.03$ & $0.85 \pm 0.03$ \\
81 & $0.99 \pm 0.03$ & $0.91 \pm 0.03$ & $0.88 \pm 0.03$ \\
90 & $0.99 \pm 0.03$ & $0.91 \pm 0.03$ & $0.91 \pm 0.03$ \\
100 & $0.99 \pm 0.03$ & $0.94 \pm 0.03$ & $0.94 \pm 0.03$ \\
121 & $1.00 \pm 0.03$ & $0.97 \pm 0.03$ & $0.99 \pm 0.03$ \\
141 & $1.01 \pm 0.03$ & $1.01 \pm 0.03$ & $1.04 \pm 0.03$ \\
150 & $1.03 \pm 0.03$ & $1.03 \pm 0.03$ & $1.06 \pm 0.03$ \\
\hline
\end{tabular}

3.4 Transfer of calibration coefficients from SSDL to the clinic

Simulated beam quality correction factors $k_{Q_{1}, Q_{0}}$ are shown in table 3 for the reference beam qualities $Q_{0}(\mathrm{RQR} 5)$ at $\mathrm{SSM}$ and $Q_{1}(70 \mathrm{kV}$, extra filtration $0.0 \mathrm{~mm} \mathrm{Cu})$ at the clinic. Values are listed for conductive layer thicknesses of 10, 15 and $20 \mathrm{~nm}$. Absolute values of simulated calibration coefficients are also given. 
Table 3: Simulated beam quality correction factors $k_{Q_{1}, Q_{0}}$ and simulated calibration coefficients $N_{P_{K A}, Q_{0}}^{r e f}$ and $N_{P_{K A}, Q_{1}}^{r e f}$ for conductive layer thicknesses of 10,15 and $20 \mathrm{~nm}$.

\begin{tabular}{llll}
\hline Thickness (nm) & $k_{Q_{1}, Q_{0}}$ & $N_{P_{K A}, Q_{0}}^{r e f}\left(\mathrm{Gym}^{2} \mathrm{C}^{-1}\right)$ & $N_{P_{K A}, Q_{1}}^{r e f}\left(\mathrm{Gym}^{2} \mathrm{C}^{-1}\right)$ \\
\hline 10 & & 1629 & 1617 \\
15 & 0.993 & 1341 & 1328 \\
20 & 0.990 & 1144 & 1131 \\
\hline
\end{tabular}

\subsection{Error in calibration coefficients used by the built-in KAP meter}

The presented method for the determination of more accurate $P_{K A}$ values was applied on measurements using the Siemens Axiom Aristos FX x-ray stand. The more accurate $P_{K A}$ values were compared to values reported by the built-in KAP meter. This ratio was used to determine the relative difference between the more accurate calibration coefficient and the calibration coefficient of the built-in KAP meter according to equation (14). Values of $\delta$ as a function of tube voltage for considered beam filtrations are shown in figure 8 .

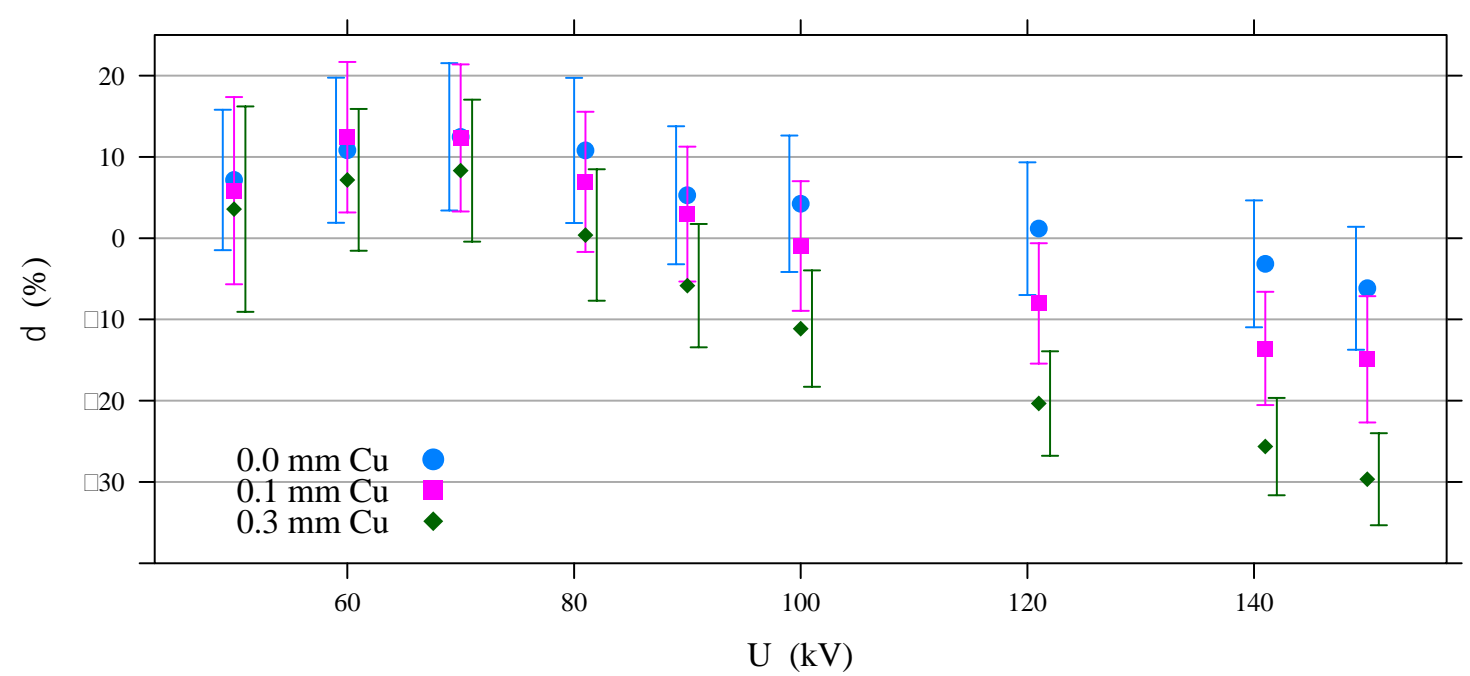

Figure 8: Relative error $\delta$ of the $P_{K A}$ value reported by the built-in KAP meter as a function of tube voltage, $U$, for filtrations of $0.0,0.1$, and $0.3 \mathrm{~mm}$ of $\mathrm{Cu}$. Error bars showing uncertainties $(\mathrm{k}=2)$ are adjusted in the horizontal direction to avoid overlapping.

Uncertainties in $\delta$ were calculated according to the GUM: Relative uncertainty of the corrected KAP meter value was assumed to be $7 \%(\mathrm{k}=2)$. Minimum relative uncertainty of the internal KAP meter reading was estimated to be $2 \%(\mathrm{k}=2)$; this value was increased for low $P_{K A}$ values owing to the fact that these values were displayed with one decimal digit only. Though the resulting values of $(-26 \pm 6) \%$ and $(-30 \pm 6) \%$ for 140 and $150 \mathrm{kV}$, respectively, and $0.3 \mathrm{~mm} \mathrm{Cu}$ exceeded the limit of $25 \%$ defined by IEC, the associated uncertainty was too large to consider these differences statistically significant at the $95 \%$ confidence level. 


\section{Discussion}

4.1 Different geometries in the standard laboratory and at measurements in the clinic The calibration coefficients are strictly valid only for the irradiation geometry used at the calibration. In the clinic, scattered radiation from materials in the beam (built-in KAP meter, collimator, plastic sheets at the end of the collimator) modifies the beam quality both regards energy spectrum as well as directions of motion of the photons incident on the reference KAP meter (see, e.g., (Malusek and Alm Carlsson 2011)) compared to the calibration at the standard laboratory. In this work, this was considered to be a second order effect and not further noted in the method of transferring the calibration coefficient from the standard laboratory to the clinic.

4.2 Choice of reference beam qualities at the standard laboratory and in the clinic To reduce the uncertainty in the transfer of the calibration coefficient from the standard laboratory to the clinic, the reference beam quality $Q_{0}$ at the standard laboratory and the reference beam quality $Q_{1}$ at the clinic should be as close to each other as possible. In this situation, uncertainties in the calculated SpekCalc spectra do not adversely affect the resulting uncertainty of $k_{Q_{1}, Q_{0}}$ for the following reason. The relative uncertainty $u\left(k_{Q_{1}, Q_{0}}\right) / k_{Q_{1}, Q_{0}}$ given by equation (20) depends on uncertainties $u\left(N_{P_{K A}, Q_{0}}\right)$ and $u\left(N_{P_{K A}, Q_{1}}\right)$ and the correlation coefficient $\rho\left(N_{P_{K A}, Q_{0}}, N_{P_{K A}, Q_{1}}\right)$. Differences between true spectra and spectra calculated with SpekCalc contribute the same to the calibration coefficients $N_{P_{K A}, Q_{0}}$ and $N_{P_{K A}, Q_{1}}$ and therefore the correlation coefficient in equation (20) is positive. On the other hand for different $Q_{0}$ and $Q_{1}$, the bias of the SpekCalc spectra may contribute differently to the calibration coefficients and thus the correlation coefficient may not be positive. This will increase the relative uncertainty $u\left(k_{Q_{1}, Q_{0}}\right) / k_{Q_{1}, Q_{0}}$.

It is noted that the RQR and RQA qualities do not provide any $\mathrm{Cu}$ filtered beams. In view of the frequent use of heavy $\mathrm{Cu}$ filtrations, in particular used in fluoroscopy, it would be worth investigating whether standard laboratories should provide $\mathrm{Cu}$ filtered reference beams to reduce uncertainties of the transfer process.

\subsection{Uncertainty analysis}

To test the possibility of limiting uncertainties of values of the air kerma-area product to $\pm 7 \%$ $(\mathrm{k}=2)$, uncertainties in the transfer of the calibration coefficient from the calibration laboratory to the clinical beams must be accurately evaluated as well as the uncertainty in properly accounting for the energy dependence of the built-in KAP meter. An in-depth analysis of all factors contributing to the total uncertainty will be needed and was beyond the scope of the present work.

A less complicated uncertainty analysis would result if the energy dependence of both the built-in KAP meter and the reference KAP meter could be reduced. This could be achieved using chambers equipped with conductive layers of, e.g., carbon (or another air equivalent material). However, such chambers are not transparent to light, which is required when using light for indicating the collimator settings. The need for light transparency does not apply to the reference chamber. There is one commercially available KAP meter (the RadCal patient dose calibrator) with a comparatively low energy dependence that could provide a better performance as a reference KAP meter (Toroi et al 2009).

\subsection{Notes on the accuracy of the beam area $A$}

Larsson et al (1996) investigated accuracy of the calibration method based on the approximation in equation (11). They found that inaccuracies in determining the nominal beam area $A$, mainly caused by a lack of widely accepted calibration procedures at that time, lead to large uncertainties 
in reported calibration coefficient. As a result of IAEA's efforts, recommendations on KAP meter calibration procedures are now widely recognized. However problems with inaccuracies arising from the approximation of the integral $\int K_{\text {air }} d A$ with the product $K_{\text {air }} A$ (beam-area method), where $K_{\text {air }}$ is determined at a single point only, remain. The advantage of the method presented in this paper is that this approximation is used neither in its original form nor in a form where the energy dependence of the reference KAP meter is determined by measurements with an energy independent ionization chamber in clinical beams. Another advantage is that the integration area $A$, given by the sensitive surface of a KAP meter, covers a wider region than just the nominal beam area. Stray radiation hitting the patient is thus more likely accounted for by the proposed method than by the beam-area method.

Measurements of the energy dependence of the reference KAP meter (not depending on the accurate knowledge of the beam area $A$ ) were performed to check the calculated beam quality correction factors in the clinical beams. The measurements could also have been used (equation (13)) to determine the corresponding beam quality correction factors for the built-in KAP meter thus leaving the physicists with a completely experimental calibration procedure except for the transfer of the calibration coefficient from the standard laboratory to a suitable reference quality in the clinical laboratory. A practical problem may be access to a suitable energy independent ion chamber in the x-ray department. On the other hand calculated beam quality correction factors for the reference KAP meter need to be made available or, alternatively, access to a suitable model of the reference KAP meter and a software program for calculating the beam quality correction factors.

\section{Short summary and conclusions}

A method to check the calibration coefficients used by built-in KAP meters was developed based on use of a reference KAP meter and the tandem calibration method. Traceability to a primary standard was achieved by calibration of the reference KAP meter at a secondary standard laboratory. Transfer of the calibration coefficient at the standard laboratory to the clinical beams was based on Monte Carlo simulations of the reference KAP meter and energy spectra obtained using the computational program SpekCalc. It was shown that the built-in KAP meter did not account for the energy dependence of the chamber, resulting in errors in reported values of the air kerma-area product of more than $25 \%$ when used in clinical beams in the range of tube voltages $50-150 \mathrm{kV}$ and added filtrations $0.1-0.3 \mathrm{~mm} \mathrm{Cu}$. This is not acceptable if the KAP values are to be compared to the diagnostic reference levels or used for patient dose optimization, as the general consensus in the field is that reductions in patient dose of the order of $10-15 \%$ are worthy of consideration. Technical developments to achieve such reductions should be implemented if the costs are reasonable.

\section{Acknowledgment}

The authors are thankful to (i) the Swedish Radiation Safety Authority for financial support and help with the calibration of KAP meters, (ii) the County Council of Östergötland for providing its facilities, (iii) Peter Larsson for help with the measuring equipment, and (iv) Jan Lillhök for valuable comments. 


\section{Appendix}

7.1 Uncertainty of the calculated calibration coefficient.

The standard combined uncertainty $u_{c}\left(\varepsilon_{s}\right)$ of the response of a KAP meter to a polyenergetic beam (equation (1)) was estimated as

$$
u_{c}^{2}\left(\varepsilon_{s}\right)=\sum_{i=1}^{N} w^{2}\left(E_{i}\right) u^{2}\left(\varepsilon\left(E_{i}\right)\right)
$$

where $u\left(\varepsilon\left(E_{i}\right)\right)$ is the standard uncertainty of the response function at photon energy $E_{i}$. It was assumed that input quantities $\varepsilon\left(E_{i}\right)$ and $\varepsilon\left(E_{j}\right), i \neq j$, were uncorrelated since they were calculated in separate Monte Carlo runs. Uncertainty of the linearly interpolated values $\varepsilon\left(E_{i}\right)$ was calculated using GUM 1995 from uncertainties $u\left(\varepsilon\left(E_{i}^{\prime}\right)\right)$ and $u\left(\varepsilon\left(E_{i}^{\prime \prime}\right)\right)$ reported by the PENELOPE code as

$$
u^{2}\left(\varepsilon\left(E_{i}\right)\right)=\left(\frac{E_{i}^{\prime \prime}-E_{i}}{E_{i}^{\prime \prime}-E_{i}^{\prime}}\right)^{2} u^{2}\left(\varepsilon\left(E_{i}^{\prime}\right)\right)+\left(\frac{E_{i}-E_{i}^{\prime}}{E_{i}^{\prime \prime}-E_{i}^{\prime}}\right)^{2} u^{2}\left(\varepsilon\left(E_{i}^{\prime \prime}\right)\right),
$$

where the meaning of energies $E_{i}, E_{i}^{\prime}$ and $E_{i}^{\prime \prime}$ is explained in connection with equation (1). Equation (16) follows from the fact that a linear interpolation between points $\left(x_{1}, y_{1}\right)$ and $\left(x_{2}, y_{2}\right)$ can be written as a linear combination of values $y_{1}$ and $y_{2}$

$$
y=y_{1}+\frac{y_{2}-y_{1}}{x_{2}-x_{1}}\left(x-x_{1}\right)=y_{1} \frac{x_{2}-x}{x_{2}-x_{1}}+y_{2} \frac{x-x_{1}}{x_{2}-x_{1}}
$$

whose combined standard uncertainty is

$$
u_{c}^{2}(y)=\left(\frac{x_{2}-x}{x_{2}-x_{1}}\right)^{2} u^{2}\left(y_{1}\right)+y_{2}\left(\frac{x-x_{1}}{x_{2}-x_{1}}\right)^{2} u^{2}\left(y_{2}\right)
$$

where $u\left(y_{1}\right)$ and $u\left(y_{2}\right)$ are the standard uncertainties of $y_{1}$ and $y_{2}$, respectively.

The combined uncertainty of the calibration coefficient $N_{P_{K A}}$ in equation (5) was calculated using the GUM as

$$
\frac{u_{c}\left(N_{P_{K A}}\right)}{N_{P_{K A}}}=\frac{u_{c}\left(\varepsilon_{s}\right)}{\varepsilon_{s}}
$$

where $u_{c}\left(\varepsilon_{s}\right)$ were calculated from equation (15).

\subsection{Uncertainty of a ratio of two input variables}

The combined uncertainty $u_{c}(y)$ of a physical quantity whose expectation $y$ is given by the functional model $y=x_{1} / x_{2}$, where $x_{1}$ and $x_{2}$ are expectations of input quantities with uncertainties $u\left(x_{1}\right)$ and $u\left(x_{2}\right)$, respectively, is according to the GUM:

$$
\left[\frac{u_{c}(y)}{y}\right]^{2}=\left[\frac{u\left(x_{1}\right)}{x_{1}}\right]^{2}+\left[\frac{u\left(x_{2}\right)}{x_{2}}\right]^{2}-2 \frac{u\left(x_{1}\right)}{x_{1}} \frac{u\left(x_{2}\right)}{x_{2}} \varrho\left(x_{1}, x_{2}\right),
$$

where $-1 \leq \varrho\left(x_{1}, x_{2}\right) \leq 1$ is the correlation coefficient describing the relation between the two input quantities. For positively correlated input quantities, the correlation coefficient lowers the relative combined uncertainty $u_{c}(y) / y$ compared to situations when the input quantities are uncorrelated or have a negative correlation.

\section{References}

Bly, R., Jahnen, A., Järvinen, H., Olerud, H., Vassileva, J., Vogiatzi, S., 2011. European 
population dose from radiodiagnostic procedures - early results of Dose Datamed. Proceedings of the XVI NSFS conference-current challenges in radiation protection. Nordic Society for Radiation Protection, Reykjavík.

Boutillon, M., Perroche-Roux, A.M., 1987. Re-evaluation of the W value for electrons in dry air. Physics in Medicine and Biology 32, 213-219.

Cullen, D., Hubbell, J., Kissel, L., n.d. EPDL97: the Evaluated Photon Data Library, '97 Version, 1997. University of California, Lawrence Livermore National Laboratory: Livermore, CA.

EC, 1997. On health protection of individuals against the dangers of ionizing radiation in relation to medical exposure (Council Directive 97/43/EURATOM). European Commission.

Hubbell, J., Seltzer, S., 2004. Tables of X-ray mass attenuation coefficients and mass energyabsorption coefficients (version 1.4). National Institute of Standards and Technology, Gaithersburg, MD.

IAEA, 2007. Dosimetry in diagnostic radiology : an international code of practice. (TRS No. 457). (Vienna: International Atomic Energy Agency)

ICRU, 2005. Patient Dosimetry for X Rays used in Medical Imaging (Report 74) (No. 74). International Comission on Radiation Units \& Measurements.

IEC, 2000. Dose area product meters, IEC-60580 (No. 60580). International Electrotechnical Commission, Geneva.

JCGM, 2008. Evaluation of measurement data - Guide to the expression of uncertainty in measurement (No. JCGM 100:2008). (Joint Committee for Guides in Metrology).

Larsson, J.P., Persliden, J., Sandborg, M., Alm Carlsson, G., 1996. Transmission ionization chambers for measurements of air collision kerma integrated over beam area. Factors limiting the accuracy of calibration. Physics in Medicine and Biology 41, 2381-2398.

Malusek, A., Alm Carlsson, G., 2011. Analysis of the tandem calibration method for kerma area product meters via Monte Carlo simulations, in: Standards, Applications and Quality Assurance in Medical Radiation Dosimetry (IDOS). Presented at the International Symposium on Standards, Applications and Quality Assurance in Medical Radiation Dosimetry (IDOS), Vienna, 9-12 November 2010, IAEA, Vienna, pp. 129-136.

Nagel, H.D., 1988. Limitations in the determination of total filtration of x-ray tube assemblies. Physics in medicine and biology 33, 271.

Nowotny, R., Meghzifene, K., 2002. Simulation of the effect of anode surface roughness on diagnostic x-ray spectra. Physics in Medicine and Biology 47, 3973-3983.

NRPB, 1992. National protocol for patient dose measurements in diagnostic radiology. National Radiological Protection Board, Chilton.

Poludniowski, G., Landry, G., DeBlois, F., Evans, P.M., Verhaegen, F., 2009. SpekCalc: a program to calculate photon spectra from tungsten anode x-ray tubes. Physics in Medicine and Biology 54, N433-N438. 
RTI Electronics AB (2005), Conversion tables between HVL and Total Filtration, Application Note 1-AN-52020-11, Revision A, February 2005

Salvat, F., Fernández-Varea, J.M., 2009. Overview of physical interaction models for photon and electron transport used in Monte Carlo codes. Metrologia 46, S112-S138.

Stears, J.G., Felmlee, J.P., Gray, J.E., 1986. Half-value-layer increase owing to tungsten buildup in the X-ray tube: fact or fiction. Radiology 160, 837-838.

Toroi, P., Komppa, T., Kosunen, A., 2008. A tandem calibration method for kerma-area product meters. Physics in Medicine and Biology 53, 4941-4958.

Toroi, P., Kosunen, A., 2009. The energy dependence of the response of a patient dose calibrator. Physics in Medicine and Biology 54, N151-N156. 